\title{
Application of DNS in the regulation of link traffic flow in multi-outlet networks
}

\author{
Deng Gengsheng ${ }^{1, \mathrm{a}}$, Yan Zhihui ${ }^{1, \mathrm{~b}}$, Zou Weiping $^{1, \mathrm{c}}$, Zhou Jingjing $^{1, \mathrm{~d}}$ \\ ${ }^{1}$ Network Center, Nanchang University, Jiangxi 330000, China. \\ adgs@ncu.edu.cn, byzh@ncu.edu.cn, czwp@ncu.edu.cn, djjzhou@ncu.edu.cn
}

Key words: DNS;BIND; multi-outlet; traffic regulation

\begin{abstract}
Multi-outlet link has been widely used in the campus network. Based on the actual case, this paper introduces how to use DNS to adjust the inbound and outbound traffic of multi-outlet network, the users do not need to modify the client.According to the actual use of the outlet link, we can take the precedence to guide the flow of some of the less important or low value applications to the link of low cost traffic for the sake of improving the efficiency of the use of outlets and saving the cost.
\end{abstract}

\section{Introduction}

The campus network using a single Internet service provider (ISP) outlet link has been difficult to meet the needs of users because there are more and more campus network users with the rapid development of university information construction and network technology.Because of the objective existence of inter ISP interoperability issues, as well as the number of resources and bandwidth in the ISP own network is different, the majority of colleges and universities have adopted a multi-outlet link,For example,China Education Research Network(CERNET) and China Telecom(CTCC),China Mobile(CMCC),China Unicom(CUCC), etc[1,2,3].How to make use of multiple export link resources more reasonably and effectively and as far as possible to play the advantages of export links in the multi-outlet network environment are the problems to be considered and resolved[4,5].This article takes the example of our university to discuss how to adjust the traffic load of each link through the application of DNS in the multi-outlet network so as to optimize the quality of network service.

\section{Problem Analysis}

\subsection{Analysis of Current Situation and Problems}

There are four outlet links in our university currently, namely CERNET , CTCC, CUCC and CMCC.Users in the campus choose one of the outlet links to visit the network through routing strategy according to the purpose of visit location, when they visit the extranet resources.The resources of our university's external service are usually published in the DMZ area of CTCC.We deployed a master and a secondary DNS server in CERNET, these two DNS servers are used as the authoritative DNS analysis for the domain name of our university and the DNS resolution service tool of our campus users.

\subsubsection{Problems}

The link load is unbalanced.There is the phenomenon like part of the links has been at full capacity, but other links (assumed to link $Y$ ) traffic load is still surplus during the operation.The reason is that there are different resources abundance on different links, and therefore most of the traffic of the campus users is directed to the resource-rich link.But because there are differences in the cost of each link,the cost of the abundant resources is generally much higher.

The access speed is sometimes slow when the users vist the extranet. It is fast when visiting some sites but slow for some other sites.The reason is that the distribution of resources is in different ISP networks, the access is unblocked when the ISP link load is in low access flow, but the acess speed maybe slow when the ISP link load is in high access flow.We try to forcibly shunt the 
traffic flow of the campus network subnet $A$ to load low link $Y$ through policy routing,but there comes new problems.Even though the load of link $Y$ remains idle,there still remains the phenomenon that the access speed of the users of campus network subnet $A$ varies .The reason is that the exchange among the ISP Network visits is not smooth.If the resources the users accessed are not in link $Y$ you need to access cross-ISP.

The experience is inconsistent for extranet users when they access the the campus resources.Sometimes there comes the problem that the speed is slow when extranet users access the campus resources and even the network is inaccessible. The reason is still that the exchange among the ISP Network visits is not smooth. The resources we provided for extranet users are mainly in the CTCC DMZ zone, users meet problems when they need to access across the ISP .

\subsubsection{Situation Analysis}

According to the analysis of the current situation and problems, we can conclude the following: (1) The traffic load of multi- outlet link of campus network is uneven; (2)The exchange among the ISP Network visits is not smooth;(3) External network resources are diverse, the richness of resources in different ISP networks varies, but there are multiple link or mirroring in many large sites resources;(4) The source of extranet users is diverse,the experience is inconsistent when they access the campus resources; (5)The online experience attention is high for the users.

\subsection{Look at the issue from the perspective of DNS application}

As an important support service on the internet, DNS's function is to achieve mutual conversion between host domain name and IP addresses. When users visit a website,the domain name resolution request is firstly raised by the local DNS server set up to the user machine,after parsing into the corresponding IP address,they can finally get access to the site.The following is the result of the test by using the nslookup and ping tools in our university's CERNET environment for different DNS. As shown in Table 1,such as parsing a plurality of IP network segment lists only one, where the delay is the client to the destination IP the PING average response delay, marking (1), (2), (3) IP parsed respectively attributable to CERNET, CTCC and CMCC:

Table 1 Analysis results of different DNS server and ping average response delay under Cernet Cernet environment

\begin{tabular}{|c|c|c|c|c|c|c|}
\hline \multirow[t]{2}{*}{ Domain } & \multicolumn{2}{|c|}{$\begin{array}{l}\text { The use of Cernet } \\
\text { DNS resolution }\end{array}$} & \multicolumn{2}{|c|}{$\begin{array}{l}\text { The use of CTCC DNS } \\
\text { resolution }\end{array}$} & \multicolumn{2}{|c|}{$\begin{array}{c}\text { The use of CMCC DNS } \\
\text { resolution }\end{array}$} \\
\hline & IP & $\begin{array}{c}\text { Dela } \\
\mathrm{y}\end{array}$ & IP & $\begin{array}{c}\text { Dela } \\
\mathrm{y}\end{array}$ & IP & $\begin{array}{c}\text { Dela } \\
\text { y }\end{array}$ \\
\hline www.163.com & $\begin{array}{c}222.198 .122 .3 \\
\text { (1) }\end{array}$ & $\begin{array}{c}29 \mathrm{~m} \\
\mathrm{~s}\end{array}$ & $\begin{array}{c}218.87 .111 .250 \\
\text { (2) }\end{array}$ & $4 \mathrm{~ms}$ & $\begin{array}{c}117.169 .16 .156 \\
\text { (3) }\end{array}$ & $1 \mathrm{~ms}$ \\
\hline www.qq.com & $\begin{array}{c}115.25 .209 .39 \\
\text { (1) }\end{array}$ & $\begin{array}{c}30 \mathrm{~m} \\
\mathrm{~s}\end{array}$ & $\begin{array}{c}101.226 .103 .106 \\
\text { (2) }\end{array}$ & $26 \mathrm{~ms}$ & $\begin{array}{c}120.198 .201 .156 \\
\text { (3) }\end{array}$ & $\begin{array}{c}23 \mathrm{~m} \\
\mathrm{~s}\end{array}$ \\
\hline $\begin{array}{l}\text { www.youku.c } \\
\text { om }\end{array}$ & $\begin{array}{c}118.228 .16 .231 \\
\text { (1) }\end{array}$ & $\begin{array}{c}31 \mathrm{~m} \\
\mathrm{~s}\end{array}$ & $\begin{array}{c}116.211 .115 .227 \\
\text { (2) }\end{array}$ & $14 \mathrm{~ms}$ & $\begin{array}{c}221.181 .195 .121 \\
\text { (3) }\end{array}$ & $\underset{s}{16 m}$ \\
\hline $\begin{array}{l}\text { www.fudan.ed } \\
\text { u.cn }\end{array}$ & $\begin{array}{c}202.120 .224 .5 \\
\text { (1) }\end{array}$ & $\begin{array}{c}30 \mathrm{~m} \\
\mathrm{~s}\end{array}$ & 61.129.42.5(2) & $15 \mathrm{~ms}$ & 61.129.42.5(2) & $\begin{array}{c}15 \mathrm{~m} \\
\mathrm{~s}\end{array}$ \\
\hline
\end{tabular}

Through a simple analysis,it can be summarized as follows:

(1) When you use different ISP's DNS servers set up by users as a local DNS server, you may get different analytical results with the same domain name;(2)The resources directed by some domain names has multiple ISP network access or mirroring and they are configured with intelligent DNS resolution;(3)You can parse out the address of the ISP's network preferentially with the DNS provided by a single ISP; (4) If there is no fixed DNS, whichever address provided by the ISP will increase the load on the ISP link.

Based on the above analysis,we can find out that through different DNS settings may take effect on the destination IP the users used to access domain name,thereby affecting the link traffic. 


\section{Design and implementation of link traffic load regulation with DNS}

\subsection{Design principles and objectives}

The campus network users in our university are spread across four different campuses, the IP address of the office area cable network is assigned by CERNET, but we use the private address in the wireless network and the students apartment.The users obtain IP through DHCP.Based on the above conditions, we hope the link traffic regulation by DNS is able to achieve the following objectives ultimately:

(1) To achieve convenient accessing for extranet users when they access the campus network resources;(2) To achieve the adjustment on each link traffic of the network resources asccessed by campus network users;(3)No need to modify the client's DNS settings; (4)No need to make changes to the existing network infrastructure; (5) Be able to adjust the link traffic conveniently.

In this article, we named the traffic generated when extranet users access the campus network Inbound traffic, the corresponding traffic generated when campus network users access network resources outbound traffic.

\subsection{Design and Implementation}

The DNS servers in our university is set up under the BIND Linux platform[6], and it is used in many colleges and universities .In this articlewe discussed the problem that based on the design and implementation of link traffic load regulation with DNS under the BIND Linux platform,utilize the ACL and View (view) function to achieve the purpose that according to different source addresses , we can return to different query states or response results.

\subsubsection{Inbound link adjustment}

As the service resources provided by our university is published in the DMZ area of CTCC,therefore we deployed Nginx reverse proxy server in CERNET, CMCC, CUCC separately. We use different configuration files on differnet ISP source addresses in the authoritative DNS server BIND.Where in the CTCC profiles DNS point to CTCC DMZ zone, the remaining three domain name resolution configuration files point to the appropriate reverse proxy server, and then configure the proxy's domain name services provided on the reverse proxy server IP. When the extranet users access campus resources,DNS matchs them in turn based on the source address and returns to the corresponding ISP network address.For example if it is from CERNET, CERNET IP address will be returned, and the same for CMCC.For the sake of the users' experience, if there is no match to the corresponding ISP network, the default will be returned to CTCC IP.

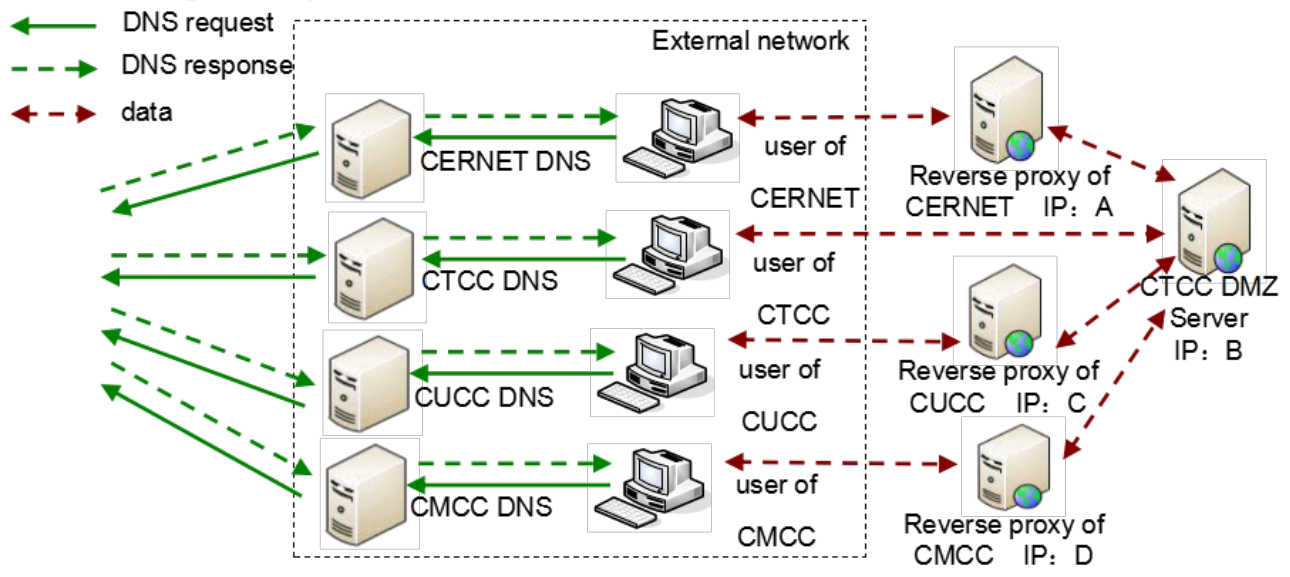

Fig.1 Inbound link regulate DNS deployment topology diagram

BIND related to the content in the main configuration are summarized below:

acl "cernet" \{

162.105.0.0/16;

\}

view "view_cernet" \{ 


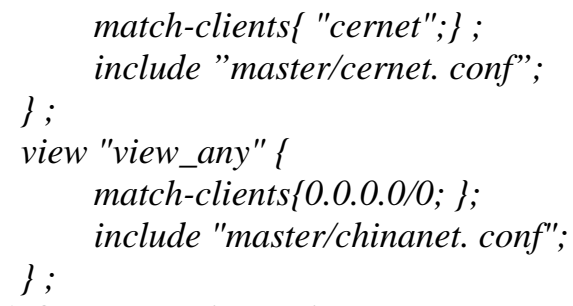

\subsubsection{Outbound link adjustment}

When the current campus network users access external resources, they will select the corresponding outlet link through policy routing based on the destination address to access external network access. Since

the campus network's CMCC outlet link is relatively abundant in the actual operation , so we directed the partial flow to the CMCC outlet link,therefore, DNS resolution resolves to the IP of CMCC preferentially.

The specific process is that we deployed the new DNS forwarding server in CTCC,CMCC, CUCC, which is used to transmit request to the DNS server provided by the operator. The original two DNS servers of CERNET are used as the primary and secondary DNS server,BIND configuration according to the rules in the user's DNS request is forwarded to the appropriate forwarding DNS server within a network deployment,the local DNS server set up for campus network users do not need to make any change, they are still the two primary and secondary DNS server. Network topologies deployed as shown in Figure 2:

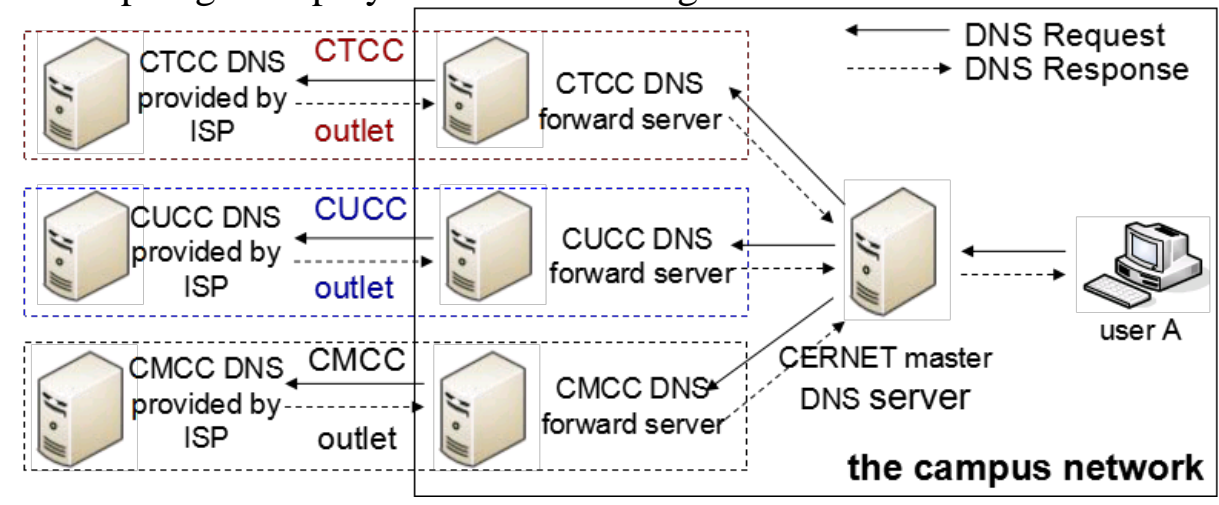

Fig. 2 Outbound link traffic load regulation DNS deployment topology

According to the features of BIND, It can be used in two ways to achieve flow regulation: (1) According to the source of IP, DNS requests will be forwarded to a different ISP network DNS servers for resolution(2) According to the domain name, DNS requests will be forwarded to a different ISP network DNS servers for resolution.Given the running situation of our university's network, we consider resoving the relatively low degree of importance which occupys a larger space's entertainment, P2P DNS domain name preferentially,give priority to the use of CMCC DNS resolution to reslove those in part of the storied building,we can make adjustment according to the chains Road traffic load.

BIND configuration content related mainly summarized as follows, in which the trust-lan-chinamobile is a list of addresses of CMCC DNS , trust-ncu section is the list of all of the addresses for the university:

include "/etc/named.conf.acls";

view "view_ncu_chinamobile" \{

match-clients \{"trust-lan-chinamobile";\};

allow-query \{"trust-lan-chinamobile";\};

forward only;

\}; forwarders $\{120.203 .222 .131 ;\}$;

view "view_ncu_any" \{

match-clients \{"trust-ncu";\};

allow-query \{"trust-ncu";\};

... 


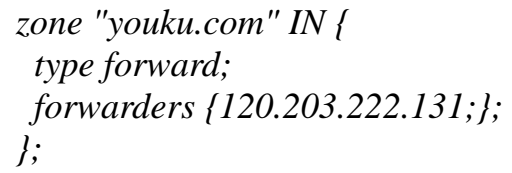

\subsubsection{Dynamic Link Load Regulation}

Since in the actual operation, the traffic load of each outlet is changed in real time,therefore we need to adopt a certain mechanism to timely adjust the configuration of the DNS link load based on the current situation.

In LINUX environments ,it can be considered to achieve the configuration file modification by using the shell command combined with timer ,the main steps are as follows: (1) Get the load of each exit from the exit device link through netsnmp, if the link are idle, exit; (2 ) Adjust the address list of users in the CERNET, CMCC, CTCC, CUCC DNS according to the situation of the load,and write them to the configuration file '/etc/named.conf.acls'; (3)check the configuration file compliance through invoking named-checkconf ; (4) If the profile is compliant, reload the configuration.

We can also use tools such as DNSTOP to count the domain names case, extract domain names with high page view and adjust the BIND configuration . Note that the match in VIEW BIND is carried out according to the order.

\section{Implementation Effect}

The CMCC outlet link traffic has been significantly increased after transmitting the cable IP address to mobile DNS preferentially for those in some of our students' dormitories. As in figure 3 which shows the DNS settings before and after the completion of a schematic flow, it was adjusted on the morning of 25th.Flow chart shows that the access traffic flow in the students' dormitories has a greater growth on that day.In the realization of the entire flow adjustment process,all the configuration only needs to be processed by the DNS server with immediate effect after the adjustment, the network or the client does not need to make any change.

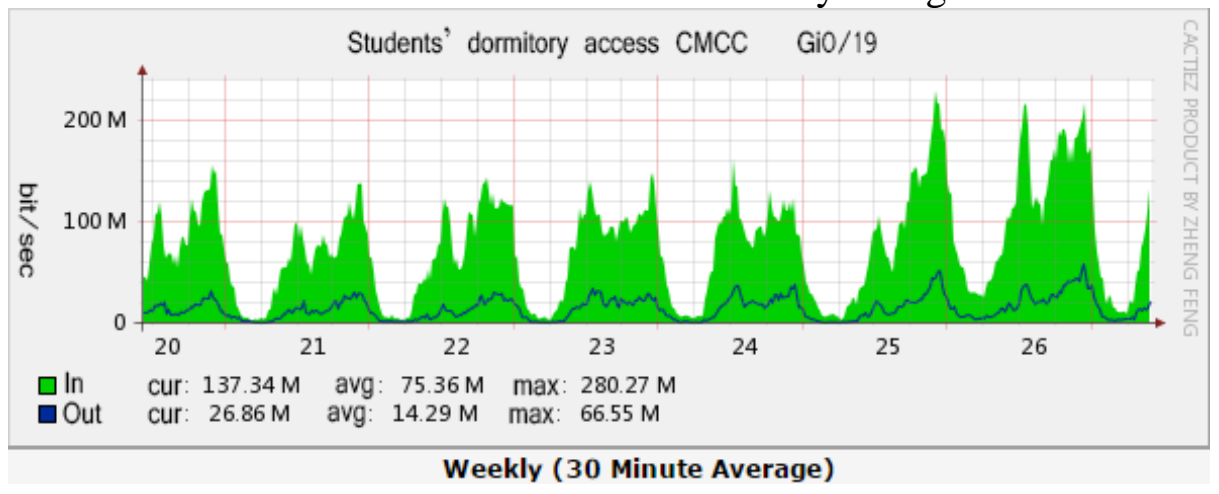

Fig. 3 Students’ dormitory cable access CMCC outlet flow diagram

\section{Summary}

This artical introduces the implementation of BIND based on DNS to adjust the inbound and outbound traffic of multi- outlet link through our university's practical cases, there is no need for the user client to make any modification.According to the application situation on the network, we can lead some of the unimportant or low value applications traffic to the links with low-flow cost to improve the use efficiency and save the the cost.We can make dynamic adjustment on the flow of the IP partition and the number of domain name through Real-time information on the traffic flow of each link and domain name access to achieve the effect of dynamic optimization link traffic load. 


\section{References}

[1] SONG Yan. Analysis and Research on the O ptim ization Allocation and Managem ent of University Network Multi-outlet Bandwidth Resources[J]. Journal of Changchun Normal University(Natural Science), 2012(3): 36-39.

[2] Sun Zheng-rong. Research and implementation of multi export load balance in campus network based on DNS service[J]. Theory and Practice ofContemporary Education, 2011(7): 173-174.

[3] Ni Ye-qing. Link equalization scheme design based on TSIG high availability DNS[J]. China Computer \& Communication, 2012(4): 96-97.

[4] CHEN Chuan-bing. Application of Intelligent DNS in traffic flow[J]. Network security technology and Application , 2014(8): 104-105.

[5] CAI Zhao-quan. Application of policy routing and dynamic DNS in campus[J]. Computer Engineering and Design, 2005(5): 1396-1398.

[6] Information on http://www.isc.org/software/bind 